\title{
Problematização sobre a pandemia da COVID-19 como auxílio na formação de enfermeiras/os
}

RESUMO | Introdução: Vivenciamos, com a Pandemia COVID-19, especulações em vários campos, com agravamentos na saúde dos indivíduos e condições econômicas dos países, principalmente daqueles vulneráveis e marginalizados nas sociedades neoliberais contemporâneas. Frente a situação, estratégias foram desenvolvidas para inserir o conteúdo COVID-19 nas disciplinas que compõe a matriz do Curso de Enfermagem do Centro Universitário São Camilo. Método: A disciplina de Vigilância em Saúde utilizou-se da abordagem por problemas. Resultado: Na realização de pesquisas, discussões em grupos e finalização das atividades propostas foram versados assuntos diversificados de interesse na formação dos estudantes para prepará-los para as atividades inerentes da Vigilância em Saúde, bem como, integrar outros temas. Conclusão: A escolha da abordagem educacional e estratégias escolhidas na Vigilância em Saúde são importantes para que nossos futuros enfermeiros possam lidar com mais precisão com as situações emergentes e reemergentes que estão e estarão pairando entre todos os locais de vivência humana.

Palavras-chaves: Aplicações da Epidemiologia; Vigilância em Saúde Pública; Educação em Enfermagem.

\begin{abstract}
I Introduction: With Pandemic COVID-19, we have experienced speculation in various fields, with deterioration in the health of individuals and economic conditions in the countries, especially those vulnerable and marginalized in contemporary neoliberal societies. Faced with the situation, strategies were developed to insert the COVID-19 content in the disciplines that make up the matrix of the Nursing Course at Centro Universitário São Camilo. Method: The discipline of Health Surveillance used the problem approach. Result: In conducting research, group discussions and finalizing the proposed activities, diversified subjects of interest in the training of students were hijacked to prepare them for the inherent activities of Health Surveillance, as well as to integrate other themes. Conclusion: The choice of the educational approach and strategies chosen in Health Surveillance are important so that our future nurses can deal more accurately with the emerging and reemerging situations that are and will be hovering between all places of human experience.
\end{abstract}

Keywords: Applications of Epidemiology; Public Health Surveillance; Nursing Education.

RESUMEN I Introducción: Con la pandemia COVID-19, estamos experimentando especulaciones en varios campos, con el empeoramiento de la salud de las personas y las condiciones económicas en los países, especialmente aquellos vulnerables y marginados en las sociedades neoliberales contemporáneas. En vista de la situación, se desarrollaron estrategias para insertar el contenido de COVID-19 en las disciplinas que conforman la matriz del Curso de Enfermería en el Centro Universitario São Camilo. Método: La disciplina de vigilancia de la salud utilizó el enfoque del problema. Resultado: al realizar investigaciones, discusiones grupales y finalizar las actividades propuestas, se diversificaron temas diversificados de interés en la capacitación de los estudiantes para prepararlos para las actividades inherentes de la Vigilancia de la Salud, así como para integrar otros temas. Conclusión: La elección del enfoque educativo y las estrategias elegidas en la Vigilancia de la salud son importantes para que nuestras futuras enfermeras puedan lidiar con mayor precisión con las situaciones emergentes y reemergentes que se ciernen y se ciernen entre todos los lugares de la experiencia humana. Palabras claves: Aplicaciones de Epidemiología; Vigilancia de Salud Pública; Educación em Enfermería.

Lourdes Bernadete dos Santos Pito A.

Doutora em Ciências pela UNIFESP; Prof ${ }^{a}$ no

Curso de Graduação em Enfermagem no Centro Universitário São Camilo.

https://orcid.org/0000-0003-4060-125X

\section{Maria Inês Nunes}

Doutora em Bioética pelo Centro Universitário São Camilo; Coordenadora do Curso de Graduação em Enfermagem do Centro Universitário São Camilo.

https://orcid.org/0000-0003-3105-6472

Recebido em: 14/05/2020

Aprovado em: 16/05/2020

INTRODUÇÃO

$\Lambda$ situação histórica que atualmente vivenciamos, com a Pandemia denominada por COVID-19, tem gerado inúmeras especulações e análises científicas, econômicas e sociais, tendo em vista a rapidez que vem atingindo a população mundial, com efeitos graves na saúde dos indivíduos e nas condições econômicas dos países e das pessoas, principalmente àqueles vulneráveis e marginalizados nas sociedades neoliberais contemporâneas.

A falta de conhecimento científico sobre tratamentos e imunobiológicos específicos tem levado a ocorrência de números significativos de infectados, de pessoas que necessitam de internação hospitalar e trata- mento intensivo, bem como, de óbitos.

Reportado pela primeira vez em dezembro de 2019 na China, o novo vírus designado de SARS-CoV-2 é o responsável pelo surto do corona vírus batizado como Covid-19 que vem causando alarme entre as autoridades sanitárias de todo o mundo e foi declarado Emergência de Saúde Pública de Importância Internacional pela Organização Mundial de Saúde (OMS) em 30 de janeiro de 20201.

Emergência de Saúde Pública de Importância Internacional (ESPII) é considerada nos termos do Regulamento Sanitário Internacional (RSI) ${ }^{2}$

“um evento extraordinário que pode constituir um risco de saúde 
pública para outros países devido a disseminação internacional de doenças; e potencialmente requer uma resposta internacional coordenada e imediata.

É a sexta vez na história que uma Emergência de Saúde Pública de Importância Internacional é declarada. As outras foram:

- 25 de abril de 2009 - pandemia de $\mathrm{H} 1 \mathrm{~N} 1$

- 5 de maio de 2014 - disseminação internacional de polio vírus

- 8 agosto de 2014 - surto de Ebola na África Ocidental

- 1 de fevereiro de 2016 - vírus Zica e aumento de casos de microcefalia e outras malformações congênitas

- 18 maio de 2018 - surto de ebola na República Democrática do Congo

A responsabilidade de se determinar se um evento constitui uma Emergência de Saúde Pública de Importância Internacional cabe ao diretor-geral da OMS e requer a convocação de um comitê de especialistas - chamado de Comitê de Emergências do RSI" ${ }^{2 \prime}$.

Em 11 de março de 2020, a COVID-19 foi caracterizada pela OMS como uma pandemia. O termo "pandemia" se refere à distribuição geográfica de uma doença e não à sua gravidade. A designação reconhece que, no momento, existem surtos de COVID-19 em vários países e regiões do mundo ${ }^{2}$.

Até o dia 12/05/2020 existiam no mundo cerca de 4.088 .848 casos confirmados; 283.153 óbitos e estes números continuam crescendo.

No Brasil, na mesma data, havia 177.589 casos confirmados e 12.400 óbitos. Comparativamente aos outros países o Brasil ocupava, neste momento, no $6^{\circ}$ posto com maior número de casos e o $6^{\circ}$ lugar em relação ao número de óbitos, muito embora tenha iniciado a transmis- são em torno de 3 meses depois de vários destes países ${ }^{3}$.

O primeiro registro de confirmação da COVID-19 no Brasil ocorreu em 26/02/20, um homem de 61 anos de São Paulo que contraiu o corona vírus em viagem à Itália, que tinha alta taxa de casos da doença. Hoje existe hipótese de que o COVID-19 já estivesse no Brasil antes desta data.

A primeira morte foi confirmada 20 dias depois, em 17 de março. O paciente era um homem de 62 anos que tinha diabetes e hipertensão. Ele estava internado na UTI do Hospital Sancta Maggiore, no bairro do Paraíso, desde o dia 14 e morreu no dia 16. Ele não tinha histórico de viagem para o exterior.

A ocorrência da pandemia denominada por COVID-19 trouxe em seu bojo vários questionamentos quanto a sua causalidade que vão além do vírus SARS-CoV-2, como:

1. teria origem no excesso de desenvolvimento tecnológico e concomitante subdesenvolvimento social, tendo em vista que populações desprovidas de acesso a informatização passam a ser marginalizadas do acesso a tudo, inclusive de capacidade de análise crítica?

2. teria a ver com a falta de harmonização com a natureza e o adentramento humano em áreas selvagens e convivência/consumo de animais inusitados?

3. ou estaria fundamentada no neoliberalismo e na globalização e a sua cruel doutrina que proclama uma drástica redução dos gastos públicos e desmantelamento da intervenção do Estado nos programas sociais, que têm sido adotados por diversos governos mundiais ${ }^{4}$ ?

Todas estas são causas plausíveis e devem ter tido influência para que ocorresse tal pandemia, mas certamente a determinação social do processo saúde-doença é a causa principal da ocorrência e rápida disseminação da doença.

Segundo Cueto (2020, sp) o neoliberalismo/globalização "criou uma cultura onde o lucro estava a cima de tudo e de todos; onde valia o corte dos recursos humanos dos sistemas de saúde, tanto nacionais quanto internacionais, e onde se banalizaram um rosário de desastres sanitários como a Aids, Dengue, SARS, H1N1, Ebola, Zika e agora a epidemia que nos oprime", criando-se, assim, um cenário propício para o desastre humanitário agora vivenciado na maior parte dos países ${ }^{5}$.

\section{MÉTODO}

Frente à situação de pandemia, estratégias foram desenvolvidas para inserir o conteúdo pertinente à COVID 19 nas unidades curriculares que compõe a matriz do curso de Enfermagem do Centro Universitário São Camilo, no caso foram escolhidas a utilização de metologias ativas como um diferencial para a formação do enfermeiro.

O curso oferece uma formação diferenciada, respaldada em uma matriz permeada por inovação e permanentemente revisada e reconstruída com foco em competências.

Nesta concepção pedagógica, merece destaque o espaço maker, metodologia realística inovadora, ambientada em Unidades Básicas de Saúde (UBS) e aplicada no $3^{\circ}$ semestre. A proposta envolve a atuação conjunta dos alunos com as equipes de Estratégia de Saúde da Família (ESF) em ações de Atenção Primária à Saúde. Acompanhados por um docente e pelo Agente Comunitário de Saúde (ACS), os alunos realizam entrevistas com a população em ambiente real: o território e comparam os resultados com os dados oficiais do Ministério da Saúde. As atividades desenvolvidas incluem: diagnóstico situacional, pesquisa, assistência de enfermagem, educação em saúde e proposta de ações para resolução de problemas.

Esta metodologia e outras servem de base para a unidade curricular de Vigilância em Saúde na qual os graduandos têm contato teórico-prático com o sistema nacional de doenças e agravos de notificação compulsória conseguem realizar a integração de conhecimento concretiza- 
dos em outras unidades curriculares, com o uso da epidemiologia descritiva e analítica para alicerçar a compreensão sobre as etapas de investigação e intervenção sobre um surto, para a compreensão de que a Vigilância em Saúde é um dos instrumentos básicos de Gestão da Saúde Coletiva como o diagnóstico coletivo em saúde e o planejamento de ações por meio dos dados epidemiológicos.

Para ser efetivado o alcance deste aprendizado são utilizadas diversas estratégias pautadas em metodologias ativas cujo eixo metodológico é a autoconstrução do conhecimento e onde os alunos desenvolverão atividades em grupos, a saber:

- Estudos de casos de doenças de notificação compulsória que são problemas de Saúde Pública na atualidade e realização de exposição do atendimento e condutas frente aos mesmos (Dengue, Sarampo, Meningite meningocócica, Febre Amarela, Tuberculose);

- Proposta de implantação de uma unidade de saúde de Estratégia de Saúde da Família (ESF) em um território do município de São Paulo, após busca e análise dos dados epidemiológicos da Atenção Básica do Município de São Paulo (boletim CElnfo);

- Seleção e/ou discussão de material da mídia escrita e eletrônica para auxiliar na compreensão sobre a investigação de surtos ${ }^{6}$ (caso COVID-19);

- Pesquisa bibliográfica Guia de Vigilância em Saúde: volume único ${ }^{7}$ do Ministério da Saúde, pesquisa nos sistemas de informação oficiais e dados secundários (SIM; SINASC, IBGE, SIH, SINAN) com a utilização da ferramenta do TABNET ${ }^{8}$.

\section{RESULTADOS}

Aprendizagem baseada em problema utilizada na unidade curricular da Vigilância em Saúde buscamos propiciar a reflexão sobre uma situação problema, ainda atual e em curso, e em todos os seus contornos, o que tem sido a abordagem educacional utilizada para buscar o alcance do preparo destes futuros enfermeiros com atributos que incluam visão sistêmica, capacidade de comunicação e negociação, habilidades para gerenciar serviços, hábito de auto aprendizagem e um olhar mais preparado para lidar com a complexidade e a incerteza, além de aprendizagem real e duradoura sobre os conceitos que cercam a Vigilância em Saúde 9 .

$\mathrm{Na}$ atual situação epidemiológica foram escolhidos como principais problemas: a pandemia da COVID-19, a epidemia de sarampo e de dengue, o controle da hanseníase, meningite meningocócica e febre amarela.

Os problemas a serem debatidos e resolvidos em grupos são o ponto de partida do processo de aprendizagem por diversos motivos: se propõe a ser um ativador dos conhecimentos prévios dos participantes do grupo sobre o tema; constitui o foco para integração do conhecimento adquirido em outras disciplinas, exercendo um papel importante na construção de estruturas cognitivas, o que facilita a recuperação e utilização posterior do conhecimento; auxilia na ressignificação das informações, fato essencial para a aprendizagem; permite um aprendizado contextualizado, no sentido que se aproxima dos problemas da vida prática, estimulando a motivação intrínseca dos alunos.

Neste caso o professor deve assumir a função como facilitador, estimulando o pensamento crítico e o auto aprendizado entre os estudantes. Para tanto o facilitador tem a função de:

- manter o fluxo das discussões;

- esquadrinhar o conhecimento do estudante profundamente, através perguntas, questionamentos, durante a análise e resolução dos problemas, estimulando o grupo a pensar crítica e profundamente sobre o tema;

- realizar questionamentos que auxiliem os estudantes a descobrir e resolver por si mesmos quaisquer possíveis erros de concepções;

- prover o grupo de informações, exemplos práticos, ou ilustrar com situações similares para estimular o aprofundamento da discussão;

- detectar os pontos positivos e negativos do funcionamento do grupo, com o objetivo de dar feedback sobre a participação e cooperação de cada alunos no grupo e sobre a qualidade do conteúdo das discussões.

Enfim, cabe ao professor: auxiliar o grupo a lidar com os problemas de relações pessoais, assegurar que todos os estudantes se envolvam de forma efetiva no processo de discussão de grupo e favorecer o desenvolvimento individual e do grupo ${ }^{8}$.

A avaliação na aprendizagem baseada em problemas deve incorporar várias estratégias, como: mapa conceitual, apresentações orais, estudos de casos, entre outros.

\section{DISCUSSÃO}

O processo de aprendizagem da Unidade Curricular de Vigilância em Saúde na Graduação em Enfermagem no Centro Universitário São Camilo em 2020 partiu da divisão dos estudantes em grupos e a divisão dos dias letivos em atividades, a saber:

- Leitura, em grupos, do Boletim Epidemiológico da Doença Respiratória de 2019-nCoV e identificação dos principais conceitos e discussão em sala de aula (conceituação de caso suspeito, emergência epidemiológica, Regulamento Sanitário Internacional, notificação compulsória, intervenções - vigilâncias epidemiológica, sanitária e ambiental, cadeia epidemiológica, etc) 6 .

- Ver o filme "Resgate Histórico de Londres: surto de doenças e óbitos por doenças respiratórias" e realizar uma reflexão e análise crítica sobre toda a contextualização do problema: momento histórico, mudança de uso de combustível, 
fatores ambientais, sobreposição de adoecimentos da influenza aos problemas cardíacos e respiratórios conectados à poluição aérea, usos da epidemiologia para organização e analise os dados. Realizar correlação com a COVID-19.

- Realizar o desenho ou montagem escrita da cadeia epidemiológica de algumas doenças mais importantes no Município de São Paulo e identificar algumas intervenções passíveis de interromper a cadeia epidemiológica das doenças.

- Ver o filme sobre etapas de investigação de surtos e fazer uma análise e correlação do que foi dito com a situação da Pandemia atual de COVID-19.

- Realizar em grupos predeterminados os estudos de casos, previamente estabelecidos, de: meningite, hanseníase, sarampo, dengue e febre amarela, outras doenças de importância em Saúde Pública na atualidade epidemiológica do país.

- Apoio Laboratorial na Vigilância em Saúde Doenças transmissíveis e a sua relevância epidemiológica: o caso COVID-19.

- Apresentação do trabalho: justificativa da indicação Unidade de Saúde da Atenção Primária baseada na análise de indicadores de saúde das diversas regiões do Município de São Paulo, com a proposta de planejamento de saúde segundo os principais problemas identificados.

Através da realização das pesquisas, discussões em grupos/alunos da sala de aula e finalização das atividades propostas foi possível tratar vários assuntos de interesse na formação dos estudantes para prepará-los para a realização das atividades de coleta, organização, análise e intervenção em saúde inerentes da Vigilância em Saúde, bem como, de integrar outros temas, a saber:

- Compreensão a respeito da teoria da Determinação Social do Processo Saúde-doença - analisando o progresso da pandemia e geração de óbitos entre aas classes sociais com menor poder de consumo e residentes na periferia de São Paulo e na região Amazônica, conforme demonstra o mapa a seguir, que revela a ocorrência de maior número de óbitos nos Distritos Administrativos da Brasilândia e da Sapopemba na periferia de São Paulo ${ }^{9}$.
- Compreensão sobre as etapas de investigação de um surto ${ }^{10}$ e tecer análise crítica sobre o momento atual, a exemplo destas colocações realizadas por alguns grupos, como o GRUPO1:

"Atualmente vemos a investigação de surtos aplicada na pandemia de COVID-19, que agora foi classificada pela OMS como uma

\section{Mapa de óbitos, segundo Distritos Administrativos, Municipio de São Paulo, 2020.}

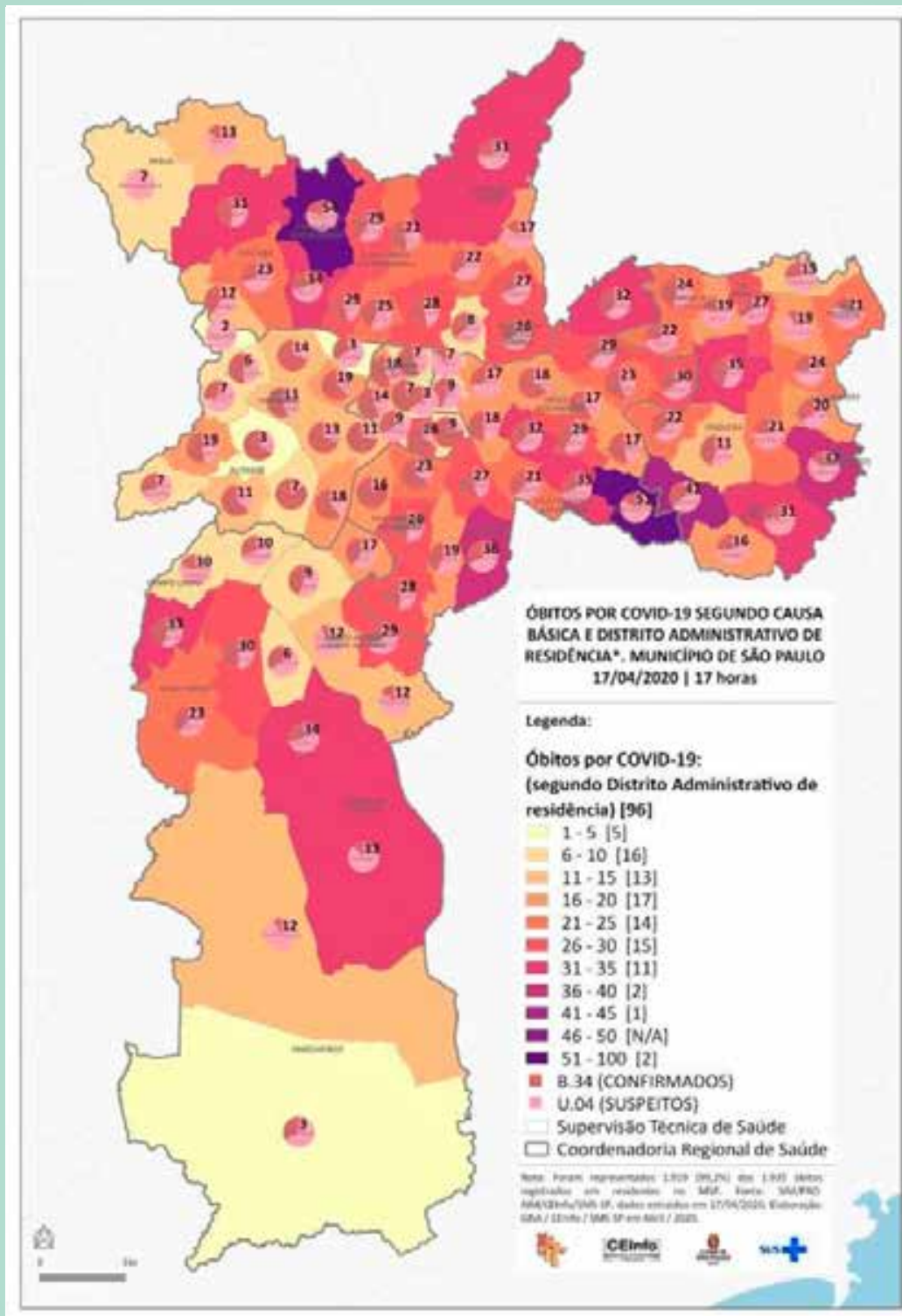


pandemia em curso, ela é uma doença respiratória aguda causada pelo novo vírus da família Corona (2019-nCoV). A doença foi identificada pela primeira vez em Wuhan, na província de Hubei, República Popular da China, em 1 de dezembro de 2019, mas o primeiro caso foi reportado em 31 de dezembro do mesmo ano. Acredita-se que o vírus tenha uma origem zoonótica, porque os primeiros casos confirmados tinham principalmente ligações ao Mercado Atacadista de Frutos do Mar de Huanan, que também vendia animais vivos.

$1^{\circ}$ Etapa: Preparar para o trabalho de campo:

Na primeira etapa é necessário realizar uma reunião preliminar. No surto de COVID-19 em dezembro do ano passado, foi solicitado reuniões para analisar as ações a serem adotadas, levando em conta os equipamentos da cidade, os aspectos administrativos. Também fizeram um levantamento de dados e referências bibliográficas para determinar o que estava acontecendo naquela cidade. Participaram os órgãos governamentais com intuito de obter (buscar) informações sobre infectados e manter a população informada sobre os dados epidemiológicos. Em 31 de dezembro de 2019, foram informados casos de pneumonia de etiologia desconhecida detectada na cidade de Wuhan, província de Hubei, para o escritório da Organização Mundial da Saúde (OMS) na China.

\section{$2^{\circ}$ Etapa: Confirmar a ocorrência da emer- gência:}

A segunda etapa tem o objetivo de confirmar se o evento é realmente uma situação inusitada para o local, região ou nação. Através da determinação dos números de casos esperados, comparando com o número de casos observados.

De 31 de dezembro de 2019 a 3 de janeiro de 2020 na China, verificaram um total de 44 pacientes com pneumonia de etiologia desconhecida, sendo notificados à OMS pelas autoridades nacionais da
China. Durante esse período relatado, o agente causal não foi identificado.

Só em 7 de janeiro de 2020, as autoridades chinesas identificaram um novo tipo de Corona vírus, que foi isolado. E em 11 e 12 de janeiro de 2020, a OMS recebeu mais informações detalhadas, da Comissão Nacional de Saúde da China, de que o surto estava associado a exposições em um mercado de frutos do mar, na cidade de Wuhan.

\section{$3^{\circ}$ Etapa: Verificar o diagnóstico}

A terceira etapa tem o objetivo de descartar os casos classificados erroneamente, por diagnósticos clínicos, laboratoriais, preenchimento etc. Revisando todas as fichas de investigação e prontuários clínicos. E entrevistando os profissionais de saúde que atenderam os pacientes, assim como os próprios pacientes e parentes. E depois padronizar e classificar o quadro clínico.

Para o surto de COVID-19, foi definido que o quadro clínico era principalmente respiratório, como por exemplo: febre, tosse e dificuldade para respirar, entre outros sintomas gripais. Pois no início, foi verificado em Wuhan, na China que muitos dos pacientes com surtos de doenças respiratórias causados por 2019-nCov tinham alguma ligação com um grande mercado de frutos do mar e animais vivos, sugerindo que a disseminação ocorreu de animais para pessoas. No entanto, um número crescente de pacientes supostamente não teve exposição ao mercado de animais, indicando também a ocorrência de disseminação de pessoa para pessoa.

As autoridades chinesas relatam que a disseminação sustentada de pessoa para pessoa estava ocorrendo na China.

\section{$4^{\circ}$ Etapa: Identificar e contar os casos}

A quarta etapa tem como função identificar novos casos, realizar a contagem total de confirmados, óbitos e suspeitos de COVID 19.

O quadro de internação por Síndrome Respiratória Aguda Grave (SRAG), aumentou cerca de $277 \%$ em 2020.

Para realização de controle e quantidade dos casos os pacientes com sinais gripais e dificuldades respiratórias são submetidos a procedimento laboratorial (PCR - Proteína C-reativa) que realiza a detecção do Corona vírus é solicitado junto à realização da Tomografia Computadorizada, pois a mesma em primeiro instante demonstra as características do pulmão, apresentado o achado "Vidro fosco", paciente com alteração no exame de imagem e com alterações respiratórias permanece em internação para controle do quadro. Os pacientes que se encontram com sinais gripais, mas sem alteração respiratória permanecem em quarentena por 14 dias aguardando resultado do exame, não sendo necessária a realização de internação.

Para controle dessa pandemia se faz necessário a realização do preenchimento da "FICHA DE NOTICAÇÃO COMPULSORIA", pois ela auxilia na contagem e no controle do vírus. Realizar padronizar a identificação dos novos casos, excluírem aqueles que não são casos, identificar o maior número possível de casos suspeitos e monitorar resultados.

\section{$5^{\circ}$ Etapa: Organizar as informações}

A quinta etapa tem como objetivo explicar como e porque o surto aconteceu. Inicialmente os habitantes de Wuhan, na província de Hubei, República Popular da China foram os primeiros afetados. Verificaram que o vírus afetava mais a população idosa acima dos 60 anos e aquelas com doenças crônicas, como diabetes e doenças cardiovasculares.

Um surto ou epidemia é uma concentração de novos casos no tempo. A existência de uma epidemia é reconhecida por uma curva epidêmica.

A exemplo da mostra dos gráficos atuais de casos novos e óbitos da COVID-19 no Brasil ${ }^{3}$.

\section{$6^{\circ}$ Etapa: Formulação de hipóteses}

O COVID-19 iniciou do China especificamente na cidade de Wuhan, acredita-se que a origem da contaminação se deu pelo Mercado Atacadista de Frutos do Mar de Huanan, constando o primeiro caso dia 31 de dezembro de 2019. Iniciaram-se então 
diversos estudos para mapear o genoma do vírus, inicialmente no começo do surto, os cientistas chineses isolaram o COVID-19, e perceberam que era $70 \%$ semelhante na sequência genética ao SARS-CoV, e posteriormente mapearam e disponibilizaram a sua sequência genética.

\section{$7^{\circ}$ Etapa: Testar as Hipóteses}

O teste destas hipóteses pode ser feito através da epidemiologia analítica: Estudo de coorte; estudo caso-controle, utilizando a estatística que é uma ferramenta.

$8^{\circ}$ Etapa: Planejar e desenvolver estudos adicionais

Através dos achados, às vezes é necessário complementar o estudo através de métodos como: Estudo epidemiológico ou estudos adicionais (ambiental ou laboratorial). No caso do COVID-19, atualmente estão sendo realizados novos estudos a cerca da profilaxia medicamentosa para este vírus, assim como estudos e teste para uma vacina.

9Etapa: Implementar medidas de controle

A nona etapa tem como objetivo controlar a fonte do organismo patogênico, interromper a transmissão e controlar a resposta de hospedeiro á exposição.

Uma das medidas para conter a propagação do vírus foi o isolamento social, sendo determinado pelos Governadores, realizado o fechamento de ambientes que promovem aglomerações, como estádios, shoppings e ambientes de lazer em geral. Permanecendo

\section{Casos novos de COVID-19 por data de notificação, Brasil, até 11/05/20.}

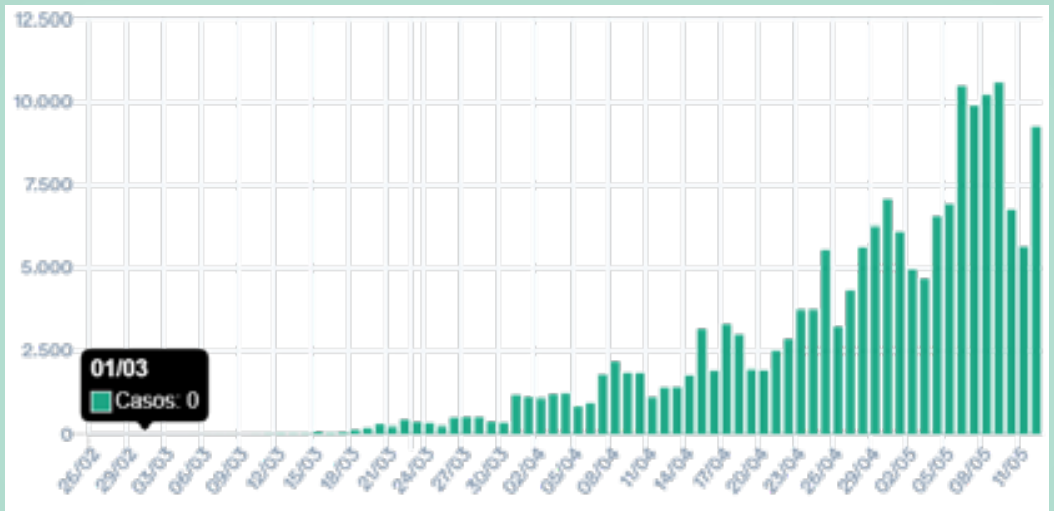

Fonte: Ministério da Saúde

\section{Óbitos de COVID-19 por data de notificação, Brasil, até 11/05/20.}

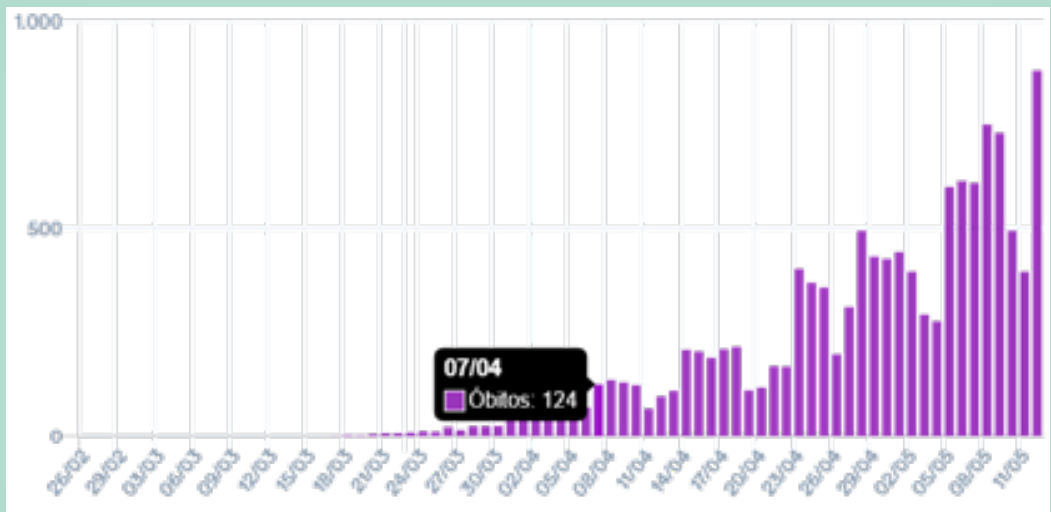

Fonte: Ministério da Saúde

abertos supermercados, farmácias, fornecedores de alimentos por delivery e fabricantes relacionados ao comercio alimentício, levando em conta todas as medidas de prevenção previamente orientadas pelos governadores e entidades de saúde.

E a higienização das mãos com água e sabão, como um dos principais meios de se evitar a transmissão. Quando não é possível realizar a lavagem das mãos com água e sabão é necessário higienizar as mãos com álcool em gel na concentração de 70\%. E a limpeza de piso, corrimão, maçaneta e banheiros devem ser feita com álcool 70\% ou solução de água sanitária.

\section{Etapa: Comunicar os resultados}

O Ministério da Saúde junto a outros órgãos está disponibilizando informações atualizadas diariamente sobre o COVID19 através de diversos meios de comunicação como, por exemplo, relatórios, boletins diários e boletins epidemiológicos, notas e notícias em telejornais, como também utilizar os programas de entretenimento para divulgar as notícias, mídias sociais, entre outros.

A atividade final trata-se de Proposta Virtual de implantação de uma unidade básica de saúde em um território do Município de São Paulo, com ênfase na Estratégia de Saúde da Família (ESF), com base nos sistemas de informações e textos bibliográficos sobre uso da vigilância em saúde e gerenciamento na atenção básica de saúde.

Ao final do trabalho o discente deverá ter capacidade de discutir de forma crítica e reflexiva frente a teoria e realidade percebida.

O processo de construção do trabalho está sendo pautado em metodologias ativas, que são acompanhadas ao final de cada aula pelo docente. $\mathrm{O}$ trabalho está sendo discutido em grupos ao final de todas as aulas da disciplina e deve iniciar pela coleta de dados, análise dos dados da territorialização e de saúde para realização da justificativa da localização do território. Os grupos devem apresentar os dados e reflexões semanalmente ao docente, que deverá acompanhar o raciocínio para implantação de uma unidade básica de saúde, com ênfase na Uni- 
dade de Saúde da Atenção Básica ESF e estar preparado para alinhamentos e dúvidas dos grupos de trabalho.

Ao final do semestre os alunos apresentarão os trabalhos com a indicação de sugestões de planejamento de atividades para impactar positivamente os problemas de saúde pesquisados indicados pelos indicadores de saúde e complementar o aprendizado de vivência da realização do diagnóstico situacional em uma microárea de uma equipe de ESF, projeto realizado durante o $3^{\circ}$ semestre da graduação $\mathrm{O}^{11,12,13,14,15}$.

\section{CONCLUSÃO}

A escolha da abordagem educacional e estratégias escolhidas para o desenvolvimento da estrutura curricular de Vigilância em Saúde são de suma importância para que nossos futuros enfermeiros possam lidar com mais precisão com as situações emergentes e reemergentes que estão e estarão pairando entre todos os locais de vivência humana.

Todos os profissionais de saúde devem estar preparados para prestar a assistência à saúde-doença não só para os indivíduos em particular, mas, também, em relação à coletividade, buscando compreender as situações a que cada população em cada território estão submetidas e a possibilidade iminente de propagação de doenças entre localidades, tendo em vista a facilidade de locomoção e o atual comportamento da sociedade globalizada.

Independentemente do setor em que estes enfermeiros venham a trabalhar, seja na área pública ou privada; de atenção primária, secundária ou terciária; no gerenciamento, educação ou pesquisa, que tenham firme a compreensão de que não existe maior ou menor importância de trabalho devido ao tipo de tecnologia utilizada.

O mais importante é executarmos a prestação do cuidado auxiliando a construção de uma rede assistencial sólida, funcionante e com boa comunicação.

Salientamos que na centralidade de todo o processo de trabalho deve estar o usuário/cliente/paciente e é por ele que a sociedade nos vê e nos dá importância enquanto utilidade profissional.
No Brasil temos um Sistema Único de Saúde - SUS - muito importante e democrático que precisa ser fortalecido e respeitado, pois as classes sociais menos abastadas têm dependência total dele e as outras partes da sociedade fazem uso dele sem saber, como nos casos dos serviços prestados pelas vigilâncias epidemiológica, ambiental, sanitária e outras, que são responsáveis pela manutenção da qualidade dos produtos e serviços que consumimos e controle e erradicação de doenças, entre outras atividades.

A cada semestre são agregados à esta unidade curricular assuntos que afetam o processo saúde-doença da população brasileira, como o acontecido com na ocorrência do rompimento da barragem de Brumadinho, e vinculado à busca de dados, bibliografias e análise crítica sobre o fato, com a utilização da teoria e desenvolvimento do raciocínio epidemiológico, e discussão em grupos.

Os discentes mostram-se muito interessados e participativos e têm levado seus trabalhos finais para os eventos científicos da área de conhecimento.

\section{Referências}

1. Fuchs, A. Covid-19: infectologista Estevão Portela fala sobre medidas preventivas e aspectos clínicos. https://portal.fiocruz.br/noticia/covid-19-infectologista-estevao-portela-fala-sobre-medidas-preventivas-e-aspectos-clinicos. [Acessado 11/05/20].

2. OPAS. Brasil. Folha informativa - COVID-19 (doença causada pelo novo coronavírus). https://www.paho.org/bra/index.php?option=com_content\&view $=$ article\&id=6101: covid19\&ltemid $=875$. [Acessado 13/05/20]

3. Brasil. Ministério da Saúde. Ministério da Saúde. https://covid.saude.gov. brl. [Acessado 13/05/20]

4. Castells. M. Fim do milênio: era da informação: economia, sociedade e cultura. $2^{a}$ ed. São Saulo: Paz e Terra. Vol 3.2000.

5. Cuetos, M. 0 Covid-19 e as epidemias da Globalização. http://www.revistahcsm.coc.fiocruz.br/o-covid-19-e-as-epidemias-da-globalizacao/ [acessado $11 / 05 / 20]$

6. Brasil. Secretaria de Vigilância em Saúde/Ministério da Saúde. Infecção Humana pelo Novo Coronavírus (2019-nCoV). Boletim Epidemiológico n8 do Centro de Operações de Emergências em Saúde Pública | COE-nCoV. COE 09 de abril de 2020. Disponível em: https://www.saude.gov.br/images/ pdf/2020/April/09/be-covid-08-final.pdf [Acessado 11/05/20].

7. Brasil. Ministério da Saúde. Secretaria de Vigilância em Saúde. Coordenadoria Geral de Desenvolvimento de Epidemiologia em Serviços. Guia de Vigilância em Saúde: volume único [recurso eletrônico]/ Ministério da Saúde. Secretaria de Vigilância em Saúde. Coordenadoria Geral de Desenvolvimento de Epidemiologia em Serviços - $2^{\mathrm{a}}$ ed. - Brasília: Ministério da Saúde, 2019, 705 p. (http://www.portalarquivos2.saude.gov.br/images/PDF/2019/ outubro/16/Volume-Unico-2019.pdf)
8. Thomaz, E. B. A. F. et al. Conceitos e ferramentas da epidemiologia. Judith Rafaelle Oliveira Pinho (Org.) - Universidade Federal do Maranhão. UNA-SUS/UFMA. São Luís: EDUFMA, 2015.

9. Mamede, S. et al. Aprendizagem baseada em problemas: anatomia de uma nova abordagem educacional. Fortaleza: Hucitec, 2001.

10. Madalosso, G. INVESTIGAÇÃO DE SURTOS: passos e análises possiveis. In: ALEXANDRE, L. B. S. P. Epidemiologia para os serviços de saúde. São Paulo: Martinari, 2012.

11. Campinas, L. L. S. L.; Martines, W. R.V Unidades básicas de saúde. In: PEREIRA, I. L.; CHANES, M.; GLVÃO, C. R. (orgs). Gestão em saúde: tendências, inovações e perspectivas. 1 ed. . São Paulo. Centro Universitário São Camilo, 2010.

12. Santos, A.S. Gestão em atenção primária à saúde e enfermagem: reflexão e conceitos importantes. In: SANTOS, A. S.; MIRANDA, S. M. R. C. (orgs). A enfermagem na gestão em atenção primária à saúde. Barueri. São Paulo. Manole, 2007.

13. Miranda, S. M. R. C. Gerenciamento da unidade básica de saúde a experiência do enfermeiro. In: SANTOS, A. S.; MIRANDA, S. M. R. C. (orgs). A enfermagem na gestão em atenção primária à saúde. Barueri. São Paulo. Manole, 2007.

14. Alves, L. A.A. R. Perfil gerencial do enfermeiro para atuar na atenção primária à saúde. In: SANTOS, A. S.; MIRANDA, S. M. R. C. (orgs). A enfermagem na gestão em atenção primária à saúde. Barueri. São Paulo. Manole, 2007. 15. São Paulo (SP). Secretaria Municipal da Saúde. Coordenação de Epidemiologia e Informação - CEInfo.Boletim CEInfo Saúde em Dados | Ano XVIII, $n^{\circ} 18$, Junho 2019. São Paulo (SP). Secretaria Municipal da Saúde, 2019, 24p. 